\title{
Computed tomography and pathology evaluation of lung ground-glass opacity
}

\author{
HUALONG YU, SHIHE LIU, CHUANYU ZHANG, SHAOKE LI, JIANAN REN, JINGLI ZHANG and WENJIAN XU
}

Department of Radiology, Affiliated Hospital of Qingdao University, Qingdao, Shandong 266101, P.R. China

Received October 27, 2015; Accepted March 3, 2017

DOI: $10.3892 /$ etm.2018.6886

\begin{abstract}
The aim of this study was to investigate the pathogenesis of lung ground-glass opacity (GGO) and the diagnostic value of computed tomography scan for lung GGO. Computed tomography (CT) images of 106 lung GGO cases were analyzed retrospectively, and the type, location, size, structure, boundaries and surrounding lung fields were evaluated. There were 12 cases of GGO with a diameter $<1.0 \mathrm{~cm}, 36$ cases with diameter of 1.0-1.5 cm, 25 cases with diameter of 1.6-2.0 cm, 19 cases with diameter of $2.0-2.5 \mathrm{~cm}$ and 14 cases with diameter of 2.5-3.0 cm. There were 20 lesions with a round shape and 68 lesions with an oval shape. There were 56 lesions with spinous processes, 18 lesions with air bronchograms and 37 lesions with surrounding pleural indentation. The diagnosis and differential diagnosis of GGO would be improved with combined CT scan and pathology results.
\end{abstract}

\section{Introduction}

Lung ground-glass opacity (GGO) presents as a mild increase in the density of the lung on the high resolution computed tomography (HRCT), while bronchial vascular bundles were visible (1). GGO is a non-specific characteristic that may be associated with various diseases, including bronchioloalveolar carcinoma (BAC) (2). In the mediastinal window of a computed tomography (CT) scan, GGO is hardly seen or only the solid part of the lung is visible. These signs can also be shown in inflammation, edema, hemorrhage, fibrosis, cancer and multiple other diseases (3). On a CT scan, four types of GGO may be observed: Type I, which was a simple ground glass-like shadow; type II, uneven density is observed; type III, central high density is observed with peripheral burring GGO; and type IV, nodular GGO is observed (4). However, with the development of HRCT, the detection and diagnosis rate of lung GGO lesions has improved significantly (5). In this paper, a

Correspondence to: Dr Wenjian Xu, Department of Radiology, Affiliated Hospital of Qingdao University, 16 Jiangsu Road, Qingdao, Shandong 266101, P.R. China

E-mail: wenjian1_xu@163.com

Key words: ground-glass opacity, computed tomography, X-ray retrospective analysis of 106 cases was performed to compare and analyze focal GGO lesions with their corresponding pathology results, with the aim of improving diagnosis and differential diagnosis, as well as the accuracy of pre-surgery diagnosis of GGO.

\section{Materials and methods}

Participants. A total of 106 cases with clinical diagnosis and CT scan of focal GGO were recruited at the Affiliated Hospital of Qingdao University (Qingdao, China) from January 2008 to July 2014, including 74 males and 32 females. The average age was $56.0 \pm 11.1$ years, ranging from 41 to 78 years old. Among them, 43 cases showed no prior clinical symptoms, and were diagnosed at the time of routine physical examination. There were 57 cases with cough or sputum, 63 cases with chest pain or chest tightness, 28 cases with hemoptysis and 36 cases with difficulty breathing. All patients underwent pulmonary multislice spiral CT examination, and 46 patients had a chest-enhanced CT scan. Patients received surgery (lobectomy, segmentectomy or partial lung resection) to remove tumor. Prior written and informed consent was obtained from all patients and the study was approved by the ethics review board of Qingdao University.

CT scan. Philips Brilliance 64-slice CT and Philips Brilliance 256 iCT layer spiral CT scanners (Philips Medical Systems B.V., Eindhoven, The Netherlands) were used. Patients were scanned in the supine position at end-expiration. Scan range was from the apex to the base of the lung, including both sides of the chest wall and axillary. The scan parameters were as follows: Tube voltage, 120-140 kV; tube current, 50-80 mA; reconstructed slice thickness, 0.3-1 mm. A high-resolution reconstruction algorithm was used with lung window and mediastinal window, including lung window of +700 to $-700 \mathrm{HU}$ and mediastinal window of 50 to $300 \mathrm{HU}$. After the CT scan, patients were injected with non-ionic iodinated contrast medium iohexol $(350 \mathrm{mgI} / \mathrm{ml}$, $1.0-1.5 \mathrm{ml} / \mathrm{kg}$, flow rate of $3-4 \mathrm{ml} / \mathrm{sec}$ ) into the ulnar vein with a binocular high-pressure syringe for an enhanced CT scan. Patients were scanned at 25 and $75 \mathrm{sec}$ after injection, for the vascular and parenchymal phases, respectively. GGO was categorized into four types according to CT scan observation: Type I; type II III and IV, as described previously (3). 
Image analysis. The scan images were adjusted for cross-sectional, sagittal, coronal and beveled sections to evaluate the shape of the lesion and its relationship with adjacent trachea. After adjustment, the CT images were read with a double-blind method by two experienced physicians. The location, size, shape (round, oval, irregular), edges (lobulated, burring, spinous process), side surface (clear, rough, fuzzy) and surroundings (vascular convergence, pleural indentation) of lesions were analyzed with plain and enhanced CT scan. Disagreements were discussed between these two physicians to reach to an agreement.

Determination of BAC content. Resected tumor tissues were fixed in $4 \%$ neutral formaldehyde at room temperature for 24-48 $\mathrm{h}$ and embedded in paraffin. Subsequently, the tumor tissues (5- $\mu \mathrm{m}$ thick sections) were stained with hematoxylin (for 3-5 min at room temperature) and eosin (for 1-4 min at room temperature) according to routine procedure. Five fields under high magnification (x100) were randomly selected. The BAC content relative to the whole tumor was evaluated and calculated as previously described (6). The tumors were categorized into four types based on BAC content: Type I, BAC content in $\geq 90 \%$ of lesion area; type II, BAC content in 50-89\% of lesion area; type III, BAC content in $10-49 \%$ of lesion area; and type IV, BAC content of $<10 \%$ lesion area.

Statistical analysis. SPSS 17.0 software (SPSS, Inc., Chicago, IL, USA) was used to compare CT scan and pathology results with $\chi^{2}$ test analysis. Spearman rank correlation analysis was used to analyze the correlation between $\mathrm{CT}$ value and $\mathrm{BAC}$ content. $\mathrm{P}<0.05$ was considered to indicate a statistically significant difference.

\section{Results}

Image characteristics of GGO. To determine the characteristics of lung lesions, HRCT scans were performed. As shown in Table I, among the 106 cases, there were 28 lesions in the right upper lobe, 21 lesions in the right middle lobe, 15 lesions in the right lower lobe, 19 lesions in the left upper lobe, 15 lesions in the left middle lobe and 8 lesions in the left lower lobe. Of the 106 cases, there were 12 lesions with diameter $<1.0 \mathrm{~cm}, 36$ lesions with diameter of $1.0-1.5 \mathrm{~cm}, 25$ lesions with diameter of 1.6-2.0 cm, 19 lesions with diameter of $2.0-2.5 \mathrm{~cm}$ and 14 lesions with diameter of 2.5-3.0 cm. There were 20 lesions of round shape (Fig. 1A), 68 of oval shape and 18 lesions of irregular shape. There were 56 lesions with burring edge (Fig. 1B) and 32 lesions with leaf edge. There were 28 cases of air bronchogram (Fig. 1C) and 37 cases of pleural indentation surrounding the lesions (Fig. 1D). There were 16 patients with lesions of 20-55 HU in enhanced CT scan. There was no obvious necrosis or calcification in all cases.

CT classification and pathology of focal GGO. To determine the association between GGO and its pathology, GGO was categorized into four types according to CT scans: Type I is simple GGO (47 cases, as in Fig. 2A-D); type II is uneven density GGO (34 cases, as in Fig. 2E and F), type III is
Table I. Computed tomography characteristics of all cases.

\begin{tabular}{|c|c|c|}
\hline \multirow[b]{2}{*}{ Variable } & \multicolumn{2}{|c|}{ Occurrence } \\
\hline & $\mathrm{n}$ & $\%$ \\
\hline \multicolumn{3}{|l|}{ Lesion location } \\
\hline Right upper lobe & 28 & 26.4 \\
\hline Right middle lobe & 21 & 19.8 \\
\hline Right lower lobe & 15 & 14.2 \\
\hline Left upper lobe & 19 & 17.9 \\
\hline Left middle lobe & 15 & 14.2 \\
\hline Left lower lobe & 8 & 7.5 \\
\hline \multicolumn{3}{|l|}{ Lesion diameter $(\mathrm{cm})$} \\
\hline$<1.0$ & 12 & 11.3 \\
\hline $1.0-1.5$ & 36 & 34.0 \\
\hline $1.6-2.0$ & 25 & 23.6 \\
\hline $2.0-2.5$ & 19 & 17.9 \\
\hline $2.5-3.0$ & 14 & 13.2 \\
\hline \multicolumn{3}{|l|}{ Lesion shape } \\
\hline Round & 20 & 18.8 \\
\hline Oval & 68 & 64.1 \\
\hline Irregular & 18 & 16.9 \\
\hline \multicolumn{3}{|l|}{ Lesion edge } \\
\hline Burring & 56 & 52.8 \\
\hline Leaf & 32 & 30.1 \\
\hline Air bronchogram & 28 & 26.4 \\
\hline Pleural indentation & 37 & 34.9 \\
\hline
\end{tabular}

central high density with peripheral burring GGO (19 cases) and type IV is nodule GGO (17 cases, as in Fig. 2G and H). Pathology of type IV GGO revealed the tumor was solid, with no air filling, proliferation of elastic fibers and interrupted or destroyed reticular structure in the tumor. The CT findings, pathology results and statistical analysis are shown in Table II. The malignancy ratio was $68.0 \%$ in GGO type I, $61.7 \%$ in type II, $73.6 \%$ in type III and $70.5 \%$ in type IV. As shown in Table II, the malignancy ratio in GGO types III and IV was higher than that in types I and II. This finding suggested that CT scan is of clinical importance in the diagnosis of GGO.

Correlation analysis between GGOCT value and BAC content. To determine the correlation of GGO CT value and BAC content, Spearman rank correlation analysis was conducted. As mentioned earlier, the tumor categorized into four types based on BAC content, including Type I $(n=26)$, Type II $(n=24)$, Type III ( $n=16)$ and Type IV $(n=13)$ (Table III). The CT values were all negatively correlated with BAC content. Furthermore, this correlation was significant $(\mathrm{P}<0.05)$.

\section{Discussion}

GGO is a hazy, dense shadow in the lung that appears on high-resolution CT of the bronchus or pulmonary vasculature. This manifestation is non-specific and can be seen 


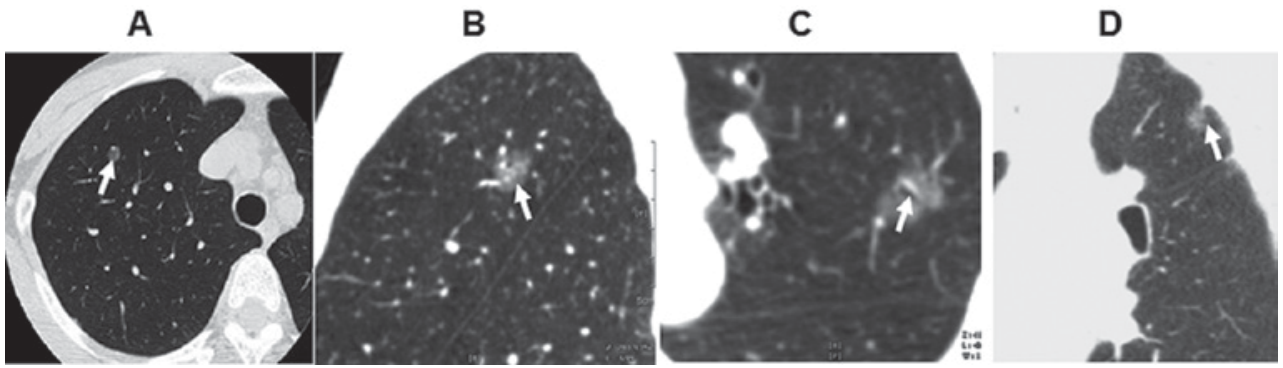

Figure 1. Examples of GGO CT imaging. (A) Nodule GGO in the right lung with round shape and no burring edges. Arrow indicated the site of the lesion. (B) Burring edge seen in sagittal section. Pathology showed BAC. Arrow indicated the burring. (C) GGO demosntrated air bronchogram (arrow) in the left upper lung in axial section. (D) GGO in the left upper lung under visceral pleura in coronal section, with surrounding pleural indentation sign (arrow). Pathology showed BAC. GGO, ground-glass opacity; CT, computed tomography; BAC, bronchioalveolar carcinoma.

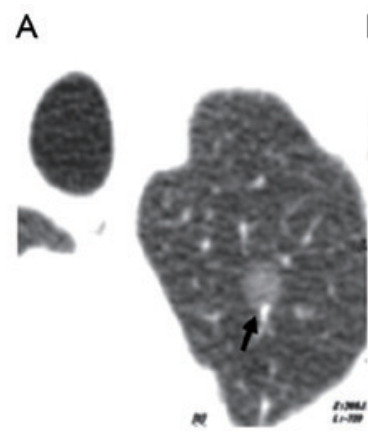

B

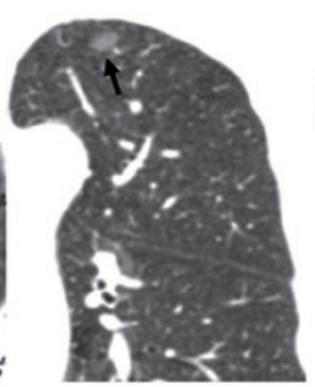

$\mathrm{F}$

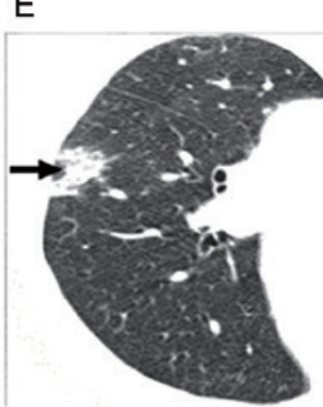

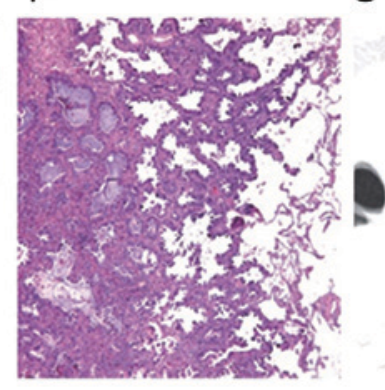

G
C
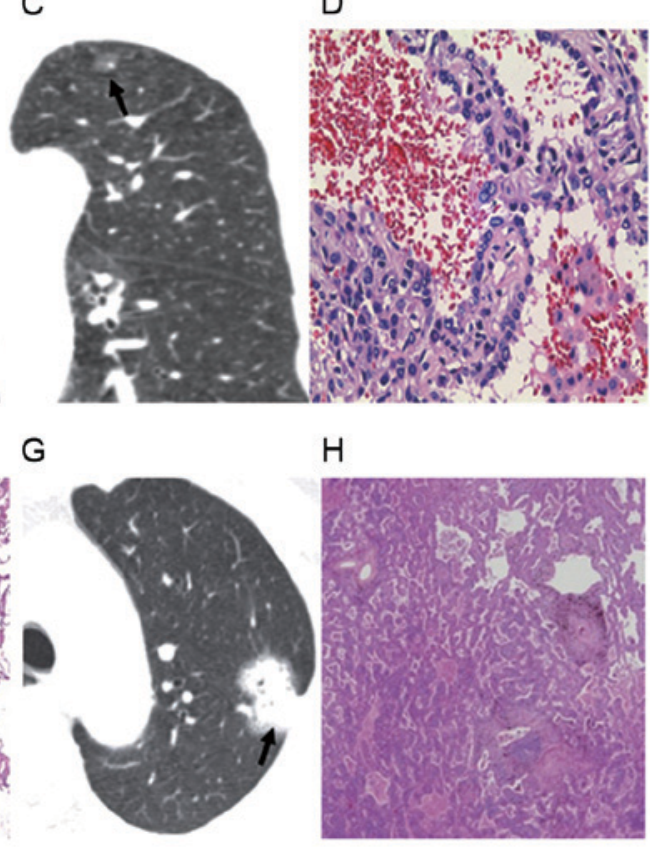

$\mathrm{H}$

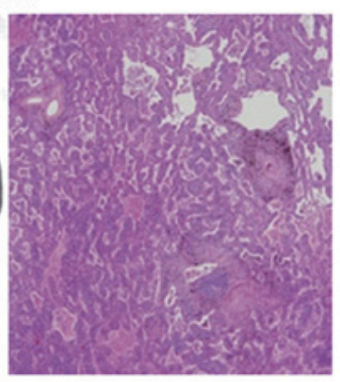

Figure 2. GGO CT classifications. (A) and (B) Simple GGO nodules in the upper lobe with clear boundary and shape, without burring. (A) GGO type IV and (B) type III were indicated. (C) Bronchial vascular bundle shown in GGO type II. (D) Pathology demonstrated incomplete filling of air cavity, mild interstitial thickening and partial alveolar depression, with pathology of BAC (GGO type I). Magnification, x100. (E) CT image showed nodules of uneven density (GGO type I). (F) Pathology indicated alveolar collapse and severe hyperplasia of elastic fibers in tumor (GGO type II). (G) CT image showed nodules of homogeneous soft tissue density (GGO type IV). Magnification, x100. (H) Pathology showed elastic fiber proliferation in tumor with interrupted and destroyed reticular structure, and pathology of lung cancer. Magnification, x100. GGO, ground-glass opacity; CT, computed tomography; BAC, bronchioalveolar carcinoma. Arrows indicate the lesions.

in inflammation, injury, edema, hemorrhage, focal fibrosis, cancer or atypical adenomatous hyperplasia (4). GGO formation results in incomplete filling of air cavity, mild interstitial thickening and partial alveolar depression (7). An increasing number of patients are clinically diagnosed with lung GGO, for unknown reasons (8). Timely detection and diagnosis of GGO is critical to future treatment and prognosis.

GGO lesions can be caused by numerous pathology changes, which generally present as incomplete filling of the alveolar cavity with cells and liquids (such as edema and hemorrhage) (9), or lung interstitial thickening due to inflammation, edema, fibrosis or cancer (10). At end-expiration, the volume of alveolar air is reduced, lung interstitial volume is normal and the number of alveolar follicles in the alveolar unit increases (10). However, with a small amount of liquid or early gas-liquid presence in alveoli, and restricted spatial resolution of high-resolution CT, it is hard to distinguish these pathology changes from the thickening of the alveolar walls (11).

In the current paper, focal GGO was divided into four types based on CT characteristics. There were 47 cases of type I, presenting as clear boundary with leaf edges or burring on CT. There were 11 cases (all type I) of peripheral GGO, with lesions below visceral pleura and away from the vascular bundle. Their pathology showed tumor cells grew along the alveolar wall with no alveolar collapse and mild hyperplasia of elastic fibers in tumor. Of the 47 cases of simple GGO, 12 cases were BAC. There were 34 cases of type II, presenting as uneven GGO on CT. Pathology showed that tumor cells grew along the alveolar wall, with scattered alveolar collapse, severe hyperplasia of elastic fibers and complete network 
Table II. Statistical analysis of CT and pathology of the 106 cases.

\begin{tabular}{lcccccr}
\hline & & & \multicolumn{2}{c}{ Pathology } & & \\
\cline { 4 - 5 } CT classification & $\mathrm{n}$ & Malignancy ratio (\%) & Benign & Malignant & $\chi^{2}$ & P-value \\
\hline I & 47 & 68.0 & 12 & 32 & 14.370 & $<0.05$ \\
II & 34 & 61.7 & 11 & 21 & & \\
III & 19 & 73.6 & 5 & 14 & & \\
IV & 17 & 70.5 & 4 & 12 & & \\
\hline
\end{tabular}

CT, computed tomography.

Table III. Correlation of different CT value and BAC content.

\begin{tabular}{lcccccr}
\hline & \multicolumn{9}{c}{ BAC content } & & \\
\cline { 2 - 5 } CT range (HU) & Type I (n=26) & Type II (n=24) & Type III (n=16) & Type IV (n=13) & r value & P-value \\
\hline-300 & 0.90 & 0.66 & 0.67 & 0.38 & -0.678 & $<0.001$ \\
-350 & 0.87 & 0.69 & 0.58 & 0.36 & -0.689 & $<0.001$ \\
-400 & 0.83 & 0.58 & 0.47 & 0.32 & -0.641 & $<0.001$ \\
-450 & 0.79 & 0.57 & 0.38 & 0.28 & -0.653 & $<0.001$ \\
-500 & 0.77 & 0.48 & 0.34 & 0.24 & -0.643 & $<0.001$ \\
-550 & 0.78 & 0.43 & 0.30 & 0.21 & -0.638 & $<0.001$
\end{tabular}

HU, Hounsfield unit; CT, computed tomography; GGO, ground-glass opacity; BAC, bronchioloalveolar carcinoma.

structure within the tumor. There were 19 cases of type III, presenting as central high-density and peripheral GGO on CT. Pathology showed alveolar collapse, proliferation of elastic fibers in tumor center, fractured reticular structure of elastic fibers and growth of tumor cells along the wall. There were 17 cases of type IV, presenting as homogeneous soft nodules on CT. Different types of GGO on CT show differences in pathology (12). In the current study, malignance accounted for $68.0 \%$ in type I, $61.7 \%$ in type II, $73.6 \%$ in type III and $70.5 \%$ in type IV. The malignance rate in types III and IV was higher than that in types I and II, which showed the clinical importance of the CT evaluation of GGO.

Lung GGO is usually associated with atypical adenomatous hyperplasia (AAH) and BAC. It has previously been reported that AAH is a precancerous lesion of lung cancer (13). This type generally consists of smaller lesions, with no leaf or burring edges on CT. In the current study, 9 cases had these characteristics. Simple GGO can also be BAC. There were 27 cases of simple GGO, but pathology showed lung cancer with pathology of tumor cell growing along the alveolar wall (14). The BAC lesions were larger than AAH, with high density, air bronchogram, burring and leaf edges, thus these two types of cancer can be differentiated by CT. Yang et al reported that out of 55 cases of BAC, $56 \%$ showed air bronchogram (15). If BAC is of peripheral type, pleural indentation may be visible (16). Solid GGO is generally shown in adenocarcinoma, which is usually larger than an AHH lesion. BAC can also show heterogeneous density with strips or patchy shadows and pleural indentation, with a smaller proportion of solid GGO compared with adenocarcinoma (17). It has been reported that mixed GGO density is of higher lung cancer incidence (18). Therefore, mixed GGO density should be considered as high-risk, and surgical intervention is highly recommended.

GGO can also be a sign of inflammation, thus regular follow-up is important to determine the nature of the lesions and further treatment. When GGO shows on a CT scan, inflammation should be ruled out first. Kodama et al (19) showed that focal GGO resulting from acute inflammation or hemorrhage can be resolved in the first three months of follow-up. If the size or density of the lesion increases within 3-6 months, it is necessary to determine the nature of the lesions. If the lesion has remained the same size or slightly increased, combined with cancer history, malignancy is of a higher possibility. If the lesion has burring or leaf edges, lung biopsy should be conducted for diagnosis. In the follow-up, regardless of the GGO size, if soft tissue has increased, adenocarcinoma is possible and surgical intervention is recommended (20). It has previously been shown that $\mathrm{CT}$ is of high specificity and moderate sensitivity in the diagnosis of focal lung nodules (21). With the development and wide application of CT examination, it can be used as the primary tool for the inspection of focal pulmonary nodules.

\section{Acknowledgements}

The authors would like to thank Professor Jizheng Lin from the Department of Radiology, Affiliated Hospital of 
Qingdao University (Qingdao, China) for his value help to this manuscript.

\section{Funding}

No funding was received.

\section{Availability of data and materials}

All data generated or analyzed during this study are included in this published article.

\section{Authors' contributions}

HLY performed the experiments, analyzed the data and wrote the manuscript. SHL, CYZ and SKL provided technical help for the experiments and were involved in drafting the manuscript. JNR and JLZ participated in acquisition of data, and, analysis and interpretation of data. WJX conceived and designed the experiments, wrote the manuscript and gave final approval of the version to be published. All authors read and approved the final manuscript.

\section{Ethics approval and consent to participate}

Prior written and informed consent was obtained from all patients and the study was approved by the ethics review board of Qingdao University.

\section{Consent for publication}

Not applicable.

\section{Competing interests}

The authors declare that they have no competing interests.

\section{References}

1. Austin JH, Müller NL, Friedman PJ, Hansell DM, Naidich DP, Remy-Jardin M, Webb WR and Zerhouni EA: Glossary of terms for CT of the lungs: Recommendations of the Nomenclature Committee of the Fleischner Society. Radiology 200: 327-331, 1996.

2. Kim HK, Choi YS, Kim J, Shim YM, Lee KS and Kim K: Management of multiple pure ground-glass opacity lesions in patients with bronchioloalveolar carcinoma. J Thorac Oncol 5: 206-210, 2010.

3. Hua Y: CT differential diagnosis of infective and non-infective diseases of the lung. Shanghai Medical Imaging 12: 337-339, 2009 (In Chinese).

4. Song Y and Zhan P: Management and differential diagnosis of ground-glass opacity pulmonary lesions. Zhonghua Jie $\mathrm{He} \mathrm{He} \mathrm{Hu}$ Xi Za Zhi 32: 808-809, 2009 (In Chinese).
5. Sergiacomi G, Cicciò C, Boi L, Velari L, Crusco S, Orlacchio A and Simonetti G: Ground-glass opacity: High-resolution computed tomography and 64-multi-slice computed tomography findings comparison. Eur J Radiol 74: 479-483, 2010.

6. Okada M, Nishio W, Sakamoto T, Uchino K, Hanioka K, Ohbayashi C and Tsubota N: Correlation between computed tomographic findings, bronchioloalveolar carcinoma component, and biologic behavior of small-sized lung adenocarcinomas. J Thorac Cardiovasc Surg 127: 857-861, 2004.

7. Engeler CE, Tashjian JH, Trenkner SW and Walsh JW: Ground-glass opacity of the lung parenchyma: A guide to analysis with high-resolution CT. AJR Am J Roentgenol 160: 249-251, 1993

8. Kobayashi Y and Mitsudomi T: Management of ground-glass opacities: Should all pulmonary lesions with ground-glass opacity be surgically resected? Transl Lung Cancer Res 2: 354-363, 2013.

9. Liu C: MSCT presentations of pulmonary ground-glass opacity. Chin Imag J Integ Trad West Med 4: 228-230, 2013 (In Chinese).

10. Bao R and Sun D: CT diagnosis and differential diagnosis of pulmonary nodular ground-glass opacity. Int J Med Radiol 31: 213-216, 2008 (In Chinese).

11. Remy-Jardin M, Giraud F, Remy J, Copin MC, Gosselin B and Duhamel A: Importance of ground-glass attenuation in chronic diffuse infiltrative lung disease: Pathologic-CT correlation. Radiology 189: 693-698, 1993.

12. Nishio R, Akata S, Saito K, Ohira T, Tsuboi M, Hirano T, Ikeda N, Kotake F, Kato $\mathrm{H}$ and Abe K: The ratio of the maximum high attenuation area dimension to the maximum tumor dimension may be an index of the presence of lymph node metastasis in lung adenocarcinomas $3 \mathrm{~cm}$ or smaller on high-resolution computed tomography. J Thorac Oncol 2: 29-33, 2007.

13. Wang J, Zhang $\mathrm{H}$, Ma X and Wu N: Atypical adenomatous hyperplasia of the lung: Correlation of radiographic and pathologic findings. Chin J Radiol 41: 483-486, 2007 (In Chinese).

14. Kakinuma R, Ohmatsu H, Kaneko M, Kusumoto M, Yoshida J, Nagai K, Nishiwaki Y, Kobayashi T, Tsuchiya R, Nishiyama $\mathrm{H}$, et al: Progression of focal pure ground-glass opacity detected by low-dose helical computed tomography screening for lung cancer. J Comput Assist Tomogr 28: 17-23, 2004.

15. Yang ZG, Sone S, Takashima S, Li F, Honda T, Maruyama Y, Hasegawa $M$ and Kawakami S: High-resolution CT analysis of small peripheral lung adenocarcinomas revealed on screening helical CT. AJR Am J Roentgenol 176: 1399-1407, 2001.

16. Liu J, Shen J, Yang C, He P, Guan Y, Liang W and He J: High incidence of EGFR mutations in pneumonic-type non-small cell lung cancer. Medicine (Baltimore) 94: e540, 2015.

17. Nambu A, Araki T, Taguchi Y, Ozawa K, Miyata K, Miyazawa M, Hiejima Y and Saito A: Focal area of ground-glass opacity and ground-glass opacity predominance on thin-section CT: Discrimination between neoplastic and non-neoplastic lesions. Clin Radiol 60: 1006-1017, 2005.

18. Fan L, Li Q, Liu S, Yu H, Li Q and Xiao X: Multi-detector computed tomography features of pulmonary mixed ground-glass opacity nodules. J Pract Radiol 27: 46-50, 2011 (In Chinese).

19. Kodama K, Higashiyama M, Yokouchi H, Takami K, Kuriyama K, Kusunoki Y, Nakayama T and Imamura F: Natural history of pure ground-glass opacity after long-term follow-up of more than 2 years. Ann Thorac Surg 73: 386-393, 2002.

20. Yun S, Shu YH and Jun GL: Investigation of High-resolution CT findings in bronchioloalveolar carcinoma. Chin J Med Imag Technol 12: 015, 2002.

21. Zhang CY, Yu HL, Li X and Sun YY: Diagnostic value of computed tomography scanning in differentiating malignant from benign solitary pulmonary nodules: A meta-analysis. Tumor Biol 35: 8551-8558, 2014. 